\title{
STATUS OF COTTON APHID, APHIS GOSSYPII GLOVER (HOMOPTERA: APHIDIDAE), RESISTANCE TO INSECTICIDES IN SOUTHERN GHANA
}

\author{
E. O. Owusu And P. M. Yeboah \\ Department of Zoology, University of Ghana, P. O. Box 67, Legon-Accra, Ghana
}

\begin{abstract}
Electrophoretic banding patterns and quantitative analysis of carboxylesterases from cotton aphid, Aphis gossypii Glover (Homoptera: Aphididae) populations infesting vegetables in southern Ghana revealed a nation-wide build-up and spread of resistant genes in most vegetable growing areas. Most aphid populations studied, especially in the Greater Accra, Ashanti and Brong Ahafo regions turned out high specific carboxylesterase activities (ranging from 16 to $53 \mathrm{nnmol} / 10 \mathrm{~min} / \mu \mathrm{g}$ protein) while complimenting with production of at least one co-migrating resistance-inducing isoenzymatic band, namely E-3, E-7, E-9 and E-10. Majority of cotton aphid populations sampled exhibited some form of insecticide resistance patterns, with high resistance occurring in certain cases. The current findings have much implications for the horticultural industry (in terms of insect pest damage control and production of residue-tainted free produce). They call for a strict national effort to regulate the influx of pesticides on the market, and ensure safe insecticide types and usage, as well as manage resistance in all concerned major insect pests.
\end{abstract}

\section{Introduction}

The use of insecticides as a management option in the control of cotton aphids, Aphis gossypii Glover (Homoptera: Aphididae) and other vegetable insect pests is on the increase in Ghana. In the case of cotton aphid, the major cause of increased insecticide application is the increasing trend of resistance in populations of the insect (Owusu, 1997). Control by known potent insecticides is met with poor results, culminating

\section{Résumé}

Owusu, O. E. \& Yеводн, M. P.: La situation de la résistance de l'aphidé de coton, Aphis gossypii Glover (Homoptera: Aphididae) aux insecticides dans le sud $d u$ Ghana. Les caractéristiques de répartition en groupes électrophorétiques et les analyses quantitatives de carboxylestérases de l'aphidé de coton, Aphis gossypii Glover (Homoptera: Aphididae), les populations infestant les légumes au sud du Ghana révélaient l'accumulation et la propagation à l'échelle nationale de gènes résistants dans la plupart de zones de la culture de légumes. Beaucoup de populations d' aphidé étudiées surtout, dans les régions de Greater Accra, Ashanti et Brong Ahafo produisaient des activités carboxylestérases spécifiques et élevées (allant de 16 à $53 \mathrm{nnmol} / 10 \mathrm{~min} / \mu \mathrm{g}$ protéin) pendant qu'elles complimentaient avec la production d' au moins une bande isoenzymatique co-migrante provoquant la résistance à savoir E-3, E-7, E-9 et E10. La majorité de populations d'aphidé de coton échantillonées donc montraient quelques formes de caractéristiques de résistance aux insecticides, avec la résistance élevée se produisant dans certains des cas. Les résultats actuels ont des implications énormes pour l'industrie horticole (sur le plan de contrôle de dégats d'insecte ravageur et la production de produit sans résidu-entaché). Ils impliquent un effort national pour régler strictement l'afflux de pesticides sur le marché, assurer que les types d' insecticide et leur utilisations sont favorables à la santé et en plus maîtriser la résistance dans tous les insectes ravageurs majeurs concernés.

in increased crop loss by farmers and backyard gardeners.

Evidence of cotton aphid insecticide resistance development in Ghana is already documented, and a call for a national policy to check the spread of resistant genes nationwide made (Owusu, 1997). Generally, insecticide resistance in cotton aphid is closely linked to both quantitative and qualitative elevation of carboxylesterase (Devonshire, 1975; Devonshire \& Moores, 1982; 
Hama \& Hosoda, 1988; Owusu et al., 1994; Owusu, Horiike \& Hirano, 1996a). Excessive production of different forms of carboxylesterase with different electrophoretic comigration band numbers and expressivities confer selective advantage to insecticide resistance (Owusu, Horiike \& Hirano, 1996a).

Activities of resistant aphid strains are much higher than those of susceptible ones, the presence of resistant-conferring comigrating bands (E-3, E-7, E-9 and E-10) in resistant strains gives a vivid qualitative difference between resistant and susceptible strains. The implication, therefore, is that selection of an isoenzyme to confer insecticide resistance may be a complex interplay of a number of factors. The prevailing esterase frequencies before the use of insecticides cannot be excluded.

In a study to ascertain the level of insecticide resistant development in aphids in Ghana, analysis of samples collected on vegetables in Accra indicated a disturbingly increasing trend of insecticide resistant (Owusu, 1997; Yeboah, 2001). Consequently, a study to assess the situation of insecticide resistance in cotton aphids was undertaken. The essence was to have an insight into the current position nation-wide, and devise a sound insecticide resistant management option to forestall the build-up and spread of resistant genes.

\section{Experimental}

Insect collection

Cotton aphids were collected from various host plants in Greater Accra, Eastern, Ashanti, Western, Volta, Central, and Brong-Ahafo regions of Ghana, and sent to Pesticide Research Laboratory, Faculty of Agriculture, Kochi University, Japan and Zoology Department, University of Ghana, on ice for analysis. Samples were analysed qualitatively and quantitatively for carboxylesterase (as an indication of insecticide resistance) by the enzymenaphthylacetate-diazo blue coupling reaction method (Devonshire, 1975; Owusu et al., 1994;
Owusu, Horiike \& Hirano, 1996a).

In-vitro assay

Quantitative assessment for carboxylesterase activities is as described previously (Owusu et al., 1994; Owusu, Kim \& Horiike, 1995; Owusu \& Adjei-Afriyie, 1996). Individual aphids were homogenized in $1 \mathrm{ml}$ of $0.1 \mathrm{M}$ potassium phosphate buffer $(p \mathrm{H} 7.0)$. Aliquots $(50 \mu \mathrm{l})$ of prepared enzymes were assayed for activity at $p \mathrm{H} 7.0$ in $2.9 \mathrm{ml}$ of buffer. Buffer-enzyme solution was incubated for $10 \mathrm{~min}$ at $40{ }^{\circ} \mathrm{C}$ with $\alpha$ naphthylacetate in ethanol as substrate. Colour development after incubation was effected with a solution mixture of sodium dodecylsulphate-fast blue B salt (SDS-FBS), and read at $600 \mathrm{~nm}$ on a spectrophotometer against a control that lacked enzyme.

\section{Electrophoresis}

Electrophoresis followed the method described by Owusu et al. (1996a, 1996b). The separating gel contained 12.2 per cent acrylamide, 0.3 per cent N, N'methylenebisacrylamide, 0.19 M Tris/ $\mathrm{HCl} p \mathrm{H} 7.8,0.07$ per cent N,N-N'N'-tetramethylethylenediamine, 0.06 per cent ammonium persulphate, and 0.2 per cent polyethylene glycol mono- $p$-octylphenyl, 1 per cent bisacrylamide, 49 $\mathrm{m} M$ Tris $/ \mathrm{HCl}(p \mathrm{H}$ 6.7) and 0.0008 per cent riboflavine. Tris/glycine buffer system was used as electrode buffer. After $2.5 \mathrm{~h}$ electrophoresis of crude enzyme preparation at $4{ }^{\circ} \mathrm{C}$, gels were dipped in $100 \mathrm{ml}$ of $0.06 M$ phosphate buffer $(p \mathrm{H} 7.0)$ containing $2 \mathrm{ml}$ of $0.03 \mathrm{M} \alpha$-naphthylacetate in acetone and $0.2 \mathrm{~g}$ of fast blue $\mathrm{B}$ or BB salt for 10 min at room temperature to visualize hydrolytic activity of isozymes.

\section{Filter paper assay}

Each individual aphid was homogenized in 20 $\mu \mathrm{l}$ of $0.06 \mathrm{M}$ phosphate buffer, $p \mathrm{H}$ 7.0. About $10 \mu \mathrm{l}$ of enzyme solution was then transferred onto filter paper, followed by a similar quantity of $30 \mathrm{~m} M \alpha-$ naphthyl acetate in ethanol. After enzyme- 
substrate reaction for about $20 \mathrm{~min}$ at $40{ }^{\circ} \mathrm{C}$, the filter paper was dipped into 0.2 per cent fast blue salt for the development of blue spots. Comparisons were then made against a standard which was graded with different concentrations of $\alpha$-naphthol in ethanol and similarly developed in fast blue salt (Owusu, Kim \& Horiike, 1995, Owusu, 1997).

\section{Results and discussion}

Cotton aphid populations collected from various locations in southern Ghana revealed wide-spread incidence of insecticide resistant genes, as indicated by both quantitative and qualitative levels of carboxylesterase (Owusu, Horiike \& Hirano, 1996ab). Apart from Kpando where majority of the insects sampled were classified as susceptible, aphids collected from all other areas were moderate to highly resistant (Tables 1-7). Samples collected from Greater Accra, Ashanti and Brong Ahafo regions were mostly classified as resistant, with rare traces of susceptible strains. This trend is not unusual considering the rate and dosage of insecticide applications in these areas. Most of the insecticides may not be for the sole purpose of cotton aphid control, but rather targeted at other major insect pests of horticultural and plantation crops (e.g. thrips, white flies, etc.), resulting in rapid spill-over on cotton aphid.

The high specific enzymatic activities, coupled with the presence of resistance-inducing comigration isoenzymatic bands E-3, E-7, E-9, and

TABLE 1

Classification of resistance in cotton aphids collected from Brong Ahafo Region

\begin{tabular}{|c|c|c|c|c|}
\hline \multirow[t]{2}{*}{ Host } & \multicolumn{3}{|c|}{ Carboxylesterase activity } & \multirow[t]{2}{*}{ Resistance level } \\
\hline & $\begin{array}{l}\text { Filter paper-visual } \\
\text { (Colour intensity)* }^{*}\end{array}$ & $\begin{array}{c}\text { Specific activity } \\
( \pm S E) \text { (nmol/ } \\
10 \mathrm{~min} / \mu \mathrm{g} \text { protein) }\end{array}$ & $\begin{array}{l}\text { Resistance-conferring } \\
\text { isoenzymatic band (s) }\end{array}$ & \\
\hline \multicolumn{5}{|l|}{ Tachiman } \\
\hline Hot pepper & ++ & $32.41 \quad(6.45)$ & E-3, E-7, & Resistant \\
\hline Garden egg & ++ & $22.71 \quad(9.56)$ & $\mathrm{E}-7$ & Moderately resistant \\
\hline Okra & ++ & $28.95 \quad(8.45)$ & E-7 & Moderately resistant \\
\hline Sweet pepper & ++ & $30.15 \quad(6.43)$ & $E-3, E-7, E-10$ & Resistant \\
\hline Cucumber & +++ & $49.21 \quad(4.78)$ & E-3, E-7, E-9, E-10 & Highly resistant \\
\hline Taro & ++ & $28.45 \quad(7.77)$ & E-7, E-10 & Moderately resistant \\
\hline \multicolumn{5}{|l|}{ Kintampo } \\
\hline Okra & ++ & $28.25 \quad(5.21)$ & $\mathrm{E}-7$ & Moderately resistant \\
\hline Hot pepper & ++ & $24.12 \quad(6.51)$ & E-3 & Moderately resistant \\
\hline Eggplant & + & $15.71 \quad(3.75)$ & Nil & Susceptible \\
\hline \multicolumn{5}{|l|}{ Sunyani } \\
\hline Hot pepper & ++ & $26.61 \quad(5.51)$ & $E-3, E-7$ & Resistant \\
\hline Eggplant & + & $18.75 \quad(4.49)$ & E-3 & Susceptible \\
\hline Water melon & ++ & $39.27 \quad(6.55)$ & E-3, E-7, E-9, E-10 & Highly resistant \\
\hline Cucumber & +++ & $52.51(10.15)$ & E-3, E-7, E-9, E-10 & Highly resistant \\
\hline
\end{tabular}

+ low activity, ++ medium activity, +++ high activity

* Reproduced from Owusu (1995). 
TABLE 2

Classification of resistance in cotton aphids collected from Volta Region

\begin{tabular}{|c|c|c|c|c|}
\hline \multirow[t]{2}{*}{ Host } & \multicolumn{3}{|c|}{ Carboxylesterase activity } & \multirow[t]{2}{*}{ Resistance level } \\
\hline & $\begin{array}{l}\text { Filter paper-visual } \\
\text { (Colour intensity)* }^{*}\end{array}$ & $\begin{array}{c}\text { Specific activity } \\
\text { ( } \pm \text { SE) (nmol/ } \\
10 \mathrm{~min} / \mu \mathrm{g} \text { protein) }\end{array}$ & $\begin{array}{l}\text { Resistance-conferring } \\
\text { isoenzymatic band (s) }\end{array}$ & \\
\hline \multicolumn{5}{|l|}{ Keta/Anloga } \\
\hline Hot pepper & ++ & $35.25(8.31)$ & E-3, E-7 & Moderately resistant \\
\hline Garden egg & + & $19.51(6.32)$ & Nil & Susceptible \\
\hline Water melon & +++ & $42.73(4.55)$ & E-3, E-7, E-9, E-10 & Highly resistant \\
\hline Eggplant & + & $24.26(7.21)$ & E-3 & Moderately resistant \\
\hline Cucumber & +++ & $46.71(9.24)$ & E-3, E-9, E-10 & Highly resistant \\
\hline Okra & ++ & $29.52(5.99)$ & E-3 & Moderately resistant \\
\hline \multicolumn{5}{|l|}{ Sogakope } \\
\hline Okra & ++ & $29.75(7.66)$ & E-3, E-7 & Moderately resistant \\
\hline Hot pepper & + & $25.25(5.79)$ & E-3 & Moderately resistant \\
\hline Eggplant & + & $16.76(4.89)$ & Nil & Susceptible \\
\hline Garden egg & + & $28.48(5.67)$ & Nil & Susceptible \\
\hline \multicolumn{5}{|l|}{ Kpando } \\
\hline Hot pepper & + & $15.25(6.42)$ & Nil & Susceptible \\
\hline Okra & + & $19.89(3.43)$ & Nil & Susceptible \\
\hline Taro & + & 14.51 (2.89) & Nil & Susceptible \\
\hline
\end{tabular}

* Refer to Table 1.

E-10, in most of the aphid populations, are irrevocably indicative of resistance development (Owusu, 1996ab). Comparing the current results in Greater Accra (Table 4) to previous ones (Owusu, 1997), it is evident that the development and spread of resistant individuals in the country is rapid and extensive. This reflects the increasing and indiscriminate use of insecticides (both dosage and application rate) on vegetable crops.

On the whole, resistance in cotton aphid can be speculated to be wide-spread in Ghana but considered to be within manageable limits (Owusu, Kim \& Horiike, 1995). There is, however, the urgent need for the formation of a national resistance management team to monitor insecticide resistance in all major insect pests, (both agriculture and medical). The problem, looks very alarming and, if not handled swiftly, the horticultural industry is likely to be hit with unpleasant crises in terms of insect pest control and pesticide residues in fruits and vegetables.

Generally, the development of resistance to pesticides by insects is appreciated as one of the most difficult steps towards effective pest control today. Nevertheless, managing resistance in cotton aphid and other arthropod pests can be approached from the view point of biological and field operational factors, which in turn lead to two categories of decision making, i.e. pesticide use and non-pesticide use. Both biological and operational factors are involved in a complex interaction that determines the overall effect of a particular chemical treatment. Consequently, proper understanding of interactions between 
TABLE 3

Classification of resistance in cotton aphids collected from Western Region

\begin{tabular}{|c|c|c|c|c|}
\hline \multirow[t]{2}{*}{ Host } & \multicolumn{3}{|c|}{ Carboxylesterase activity } & \multirow[t]{2}{*}{ Resistance level } \\
\hline & $\begin{array}{l}\text { Filter paper-visual } \\
\text { (Colour intensity)* }^{*}\end{array}$ & $\begin{array}{l}\text { Specific activity } \\
\text { ( } \pm \text { SE) (nmol/ } \\
10 \mathrm{~min} / \mu \mathrm{g} \text { protein) }\end{array}$ & $\begin{array}{l}\text { Resistance-conferring } \\
\text { isoenzymatic band (s) }\end{array}$ & \\
\hline \multicolumn{5}{|l|}{ Takoradi } \\
\hline Hot pepper & + & $20.25(5.34)$ & $\mathrm{E}-3, \mathrm{E}-7$ & $\begin{array}{l}\text { Moderately } \\
\text { resistant }\end{array}$ \\
\hline Garden egg & + & $19.15(6.66)$ & Nil & Susceptible \\
\hline Okra & ++ & $32.75(9.34)$ & $\mathrm{E}-3, \mathrm{E}-7, \mathrm{E}-10$ & Resistant \\
\hline \multicolumn{5}{|c|}{ Agona Nkwanta } \\
\hline Okra & ++ & $25.15(5.45)$ & $E-3, E-7$ & $\begin{array}{l}\text { Moderately } \\
\text { resistant }\end{array}$ \\
\hline Hot pepper & ++ & $28.15(3.98)$ & E-3 & $\begin{array}{l}\text { Moderately } \\
\text { resistant }\end{array}$ \\
\hline Cucumber & +++ & $35.75(4.77)$ & E-3, E-9, E-10 & Highly resistant \\
\hline Taro & + & $19.28(6.78)$ & Nil & Susceptible \\
\hline Eggplant & + & $18.89(3.89)$ & Nil & Susceptible \\
\hline \multicolumn{5}{|l|}{ Tarkwa } \\
\hline Hot pepper & + & $15.25(6.42)$ & E-3 & $\begin{array}{l}\text { Moderately } \\
\text { resistant }\end{array}$ \\
\hline Okra & + & $19.89(3.43)$ & E-3 & $\begin{array}{l}\text { Moderately } \\
\text { resistant }\end{array}$ \\
\hline Taro & + & $14.51(2.89)$ & Nil & Susceptible \\
\hline Eggplant & + & 10.89 & Nil & Susceptible \\
\hline
\end{tabular}

* Refer to Table 1.

these factors may be essential to devise strategies for host dependent inter and intra species complexes which occur under different ecological conditions. Thus, operational factors such as type of insecticide, dosage, time and method of application can be manipulated directly to reduce selection pressure for resistance.

Management of resistance calls for a resolution of these factorial complexities so that a better sense of judgement can be anticipated concerning the outcome of potential control strategies. Most of the pesticide resistant management considerations have stressed on relative fitness of genotypes by concepts of population genetic theories, and have failed to address options that reveal characteristics that spell out practical utilities of other alternatives. A proposed scheme for national insecticide resistance monitoring and management in aphids is currently under consideration. It is the hope that, with vigorous education on judicious pesticide usage and strict enforcement of the national pesticide policy legislature, spending millions of dollars, out of the meager national budget, to manage resistant 
TABLE 4

Classification of resistance in cotton aphids collected from Greater Accra Region

\begin{tabular}{|c|c|c|c|}
\hline \multirow[t]{2}{*}{ Host } & \multicolumn{2}{|c|}{ Carboxylesterase activity } & \multirow[t]{2}{*}{ Resistance level } \\
\hline & $\begin{array}{c}\text { Filter paper-visual Specific activity } \\
\text { (Colour intensity)* }^{*} \pm \text { SE) (nmol/ } \\
10 \mathrm{~min} / \mu \mathrm{g} \text { protein) }\end{array}$ & $\begin{array}{l}\text { Resistance-conferring } \\
\text { isoenzymatic band (s) }\end{array}$ & \\
\hline
\end{tabular}

\begin{tabular}{|c|c|c|c|c|}
\hline \multicolumn{5}{|l|}{ Accra } \\
\hline Hot pepper & ++ & $29.59(3.67)$ & $E-3, E-7$ & $\begin{array}{l}\text { Moderately } \\
\text { resistant }\end{array}$ \\
\hline Garden egg & + & $23.56(4.56)$ & $E-3, E-7$ & $\begin{array}{l}\text { Moderately } \\
\text { resistant }\end{array}$ \\
\hline Okra & ++ & $31.77(5.78)$ & E-3, E-7, E-10 & Resistant \\
\hline Cucumber & +++ & $52.19(9.87)$ & E-3, E-7, E-9, E-10 & Highly resistant \\
\hline Water melon & +++ & 48.99 (8.98) & E-3, E-7, E-9, E-10 & Highly resistant \\
\hline Eggplant & ++ & $29.87(7.89)$ & $\mathrm{E}-7, \mathrm{E}-10$ & $\begin{array}{l}\text { Moderately } \\
\text { resistant }\end{array}$ \\
\hline Taro & + & $24.89(5.78)$ & $E-3, E-7$ & $\begin{array}{l}\text { Moderately } \\
\text { resistant }\end{array}$ \\
\hline \multicolumn{5}{|l|}{ Tema } \\
\hline Okra & ++ & $27.19(5.89)$ & E-3, E-7 & $\begin{array}{l}\text { Moderately } \\
\text { resistant }\end{array}$ \\
\hline Hot pepper & ++ & $30.15(8.98)$ & E-3, E-9, E-10 & Highly resistant \\
\hline Cucumber & +++ & $45.89(10.43)$ & E-3, E-7, E-9, E-10 & Highly resistant \\
\hline Taro & + & 23.89 (6.79) & $E-3, E-7$ & $\begin{array}{l}\text { Moderately } \\
\text { resistant }\end{array}$ \\
\hline Eggplant & + & $26.15(7.79)$ & $\mathrm{E}-3, \mathrm{E}-7$ & $\begin{array}{l}\text { Moderately } \\
\text { resistant }\end{array}$ \\
\hline Water melon & +++ & $42.15(7.79)$ & E-3, E-9, E-10 & Highly resistant \\
\hline \multicolumn{5}{|l|}{ Weija } \\
\hline Hot pepper & ++ & $22.15(8.90)$ & E-3, E10 & Resistant \\
\hline Okra & ++ & $29.98(6.98)$ & E-3, E7 & $\begin{array}{l}\text { Moderately } \\
\text { resistant }\end{array}$ \\
\hline Taro & ++ & $22.16(7.95)$ & $\mathrm{E}-7$ & $\begin{array}{l}\text { Moderately } \\
\text { resistant }\end{array}$ \\
\hline Eggplant & + & $19.19(5.43)$ & E-3 & Susceptible \\
\hline Cucumber & +++ & $39.89(8.97)$ & E-3, E-7, E-9, E-10 & Highly resistant \\
\hline Sweet pepper & ++ & 31.67 (6.09) & E-7, E-9, E-10 & Highly resistant \\
\hline
\end{tabular}

* Refer to Table 1. 
TABLE 5

Classification of resistance in cotton aphids collected from Eastern Region

\begin{tabular}{|c|c|c|c|c|}
\hline \multirow[t]{2}{*}{ Host } & \multicolumn{3}{|c|}{ Carboxylesterase activity } & \multirow[t]{2}{*}{ Resistance level } \\
\hline & $\begin{array}{l}\text { Filter paper-visual } \\
\text { (Colour intensity)* }^{*}\end{array}$ & $\begin{array}{l}\text { Specific activity } \\
\text { ( } \pm \text { SE) (nmol/ } \\
10 \mathrm{~min} / \mu \mathrm{g} \text { protein) }\end{array}$ & $\begin{array}{l}\text { Resistance-conferring } \\
\text { isoenzymatic band (s) }\end{array}$ & \\
\hline \multicolumn{5}{|c|}{ New Tafo Akim } \\
\hline Hot pepper & + & $20.09(5.67)$ & E-3 & Susceptible \\
\hline Garden egg & + & $15.45(3.89)$ & E-3 & Susceptible \\
\hline Okra & ++ & 27.68 (8.98) & E-3, E-7 & Resistant \\
\hline Taro & + & 19.89 (8.97) & Nil & Susceptible \\
\hline \multicolumn{5}{|l|}{ Kade } \\
\hline Okra & ++ & 28.99 (6.79) & E-3, E-7 & Moderately resistant \\
\hline Hot pepper & ++ & $27.76(8.89)$ & E-3, E-7, E-10 & Resistant \\
\hline Garden egg & + & $21.65(3.67)$ & E-3 & Moderately resistant \\
\hline Taro & + & $23.45(4.56)$ & E-3, E-7 & Moderately resistant \\
\hline \multicolumn{5}{|c|}{ Mamfe Akuapim } \\
\hline Hot pepper & ++ & 29.98 (8.99) & $E-3, E-10$ & Resistant \\
\hline Okra & ++ & 32.17 (6.78) & $E-3, E-7, E-10$ & Resistant \\
\hline Taro & ++ & $25.56(7.56)$ & E-7, E-10 & Resistant \\
\hline Eggplant & + & $20.17(5.56)$ & E-3 & Moderately resistant \\
\hline Cucumber & +++ & $63.13(9.90)$ & E-3, E-7, E-9, E-10 & Highly resistant \\
\hline Sweet pepper & ++ & 38.91 (6.78) & E-3, E-9, E-10 & Highly resistant \\
\hline
\end{tabular}

* Refer to Table 1.

TABLE 6

Classification of resistance in cotton aphids collected from Central Region

\begin{tabular}{|c|c|c|c|c|}
\hline \multirow[t]{2}{*}{ Host } & \multicolumn{3}{|c|}{ Carboxylesterase activity } & \multirow[t]{2}{*}{ Resistance level } \\
\hline & $\begin{array}{l}\text { Filter paper-visual } \\
\text { (Colour intensity)* }\end{array}$ & $\begin{array}{c}\text { Specific activity } \\
\text { ( } \pm \text { SE) (nmol/ } \\
10 \mathrm{~min} / \mu \mathrm{g} \text { protein) }\end{array}$ & $\begin{array}{l}\text { Resistance-conferring } \\
\text { isoenzymatic band (s) }\end{array}$ & \\
\hline \multicolumn{5}{|l|}{ Kasoa } \\
\hline Hot pepper & +++ & $35.79(5.78)$ & E-3, E-9, E-10 & Highly resistant \\
\hline Garden egg & ++ & $29.45(7.89)$ & $\mathrm{E}-3, \mathrm{E}-7$ & Resistant \\
\hline Okra & ++ & $31.34(6.79)$ & E-3, E-7 & Resistant \\
\hline Cucumber & +++ & $48.98(7.99)$ & E-3, E-7, E-9, E-10 & Highly resistant \\
\hline \multicolumn{5}{|l|}{ Swedru } \\
\hline Okra & +++ & $38.98(8.76)$ & E-3, E-7, E-10 & Highly resistant \\
\hline Hot pepper & +++ & 43.21 (7.92) & E-3, E-7, E-10 & Highly resistant \\
\hline Garden egg & ++ & $23.51(6.78)$ & E-3, E-7 & $\begin{array}{l}\text { Moderately } \\
\text { resistant }\end{array}$ \\
\hline \multicolumn{5}{|l|}{ Elmina } \\
\hline Hot pepper & + & $19.71(5.21)$ & E-3 & $\begin{array}{l}\text { Moderately } \\
\text { resistant }\end{array}$ \\
\hline Okra & + & $17.89(3.23)$ & Nil & Susceptible \\
\hline Eggplant & + & $10.21(3.31)$ & Nil & Susceptible \\
\hline
\end{tabular}

* Refer to Table 1. 
TABLE 7

Classification of resistance in cotton aphids collected from Ashanti Region

\begin{tabular}{|c|c|c|c|c|}
\hline \multirow[t]{2}{*}{ Host } & \multicolumn{3}{|c|}{ Carboxylesterase activity } & \multirow[t]{2}{*}{ Resistance level } \\
\hline & $\begin{array}{l}\text { Filter paper-visual } \\
\text { (Colour intensity)* }^{*}\end{array}$ & $\begin{array}{c}\text { Specific activity } \\
( \pm S E) \text { (nmol/ } \\
10 \mathrm{~min} / \mu \mathrm{g} \text { protein) }\end{array}$ & $\begin{array}{l}\text { Resistance-conferring } \\
\text { isoenzymatic band (s) }\end{array}$ & \\
\hline \multicolumn{5}{|l|}{ Kumasi } \\
\hline Hot pepper & ++ & $31.24(5.23)$ & E-3, E-9, E-10 & Highly resistant \\
\hline Garden egg & ++ & $27.78(5.32)$ & E-3, E-7 & Resistant \\
\hline Okra & ++ & 31.37 (4.56) & E-3, E-7 & Resistant \\
\hline Cucumber & +++ & $47.51(4.21)$ & $\mathrm{E}-3, \mathrm{E}-7, \mathrm{E}-10$ & Highly resistant \\
\hline \multicolumn{5}{|l|}{ Konongo } \\
\hline Okra & +++ & $45.56(6.78)$ & E-3, E-7, E-9, E-10 & Highly resistant \\
\hline Hot pepper & +++ & $39.51(4.44)$ & E-3, E-7, E-10 & Highly resistant \\
\hline Garden egg & ++ & $28.51(3.21)$ & E-3, E-7 & Moderately resistant \\
\hline Taro & + & $19.51(2.89)$ & Nil & Susceptible \\
\hline \multicolumn{5}{|l|}{ Akumadan } \\
\hline Hot pepper & +++ & $46.79(5.21)$ & E3, E-7, E-10 & Highly resistant \\
\hline Okra & +++ & 59.89 (4.67) & E-3, E-7, E-9, E-10 & Highly resistant \\
\hline Eggplant & ++ & $32.78(8.21)$ & E-3, E-7, E-10 & Resistant \\
\hline Cucumber & +++ & $64.89(4.56)$ & E-3, E-7, E-9, E-10 & Highly resistant \\
\hline Sweet pepper & +++ & $56.78(3.45)$ & E-3, E-7, E-9, E-10 & Highly resistant \\
\hline Garden egg & ++ & $43.23(4.56)$ & E-3, E-7, E-9, E-10 & Highly resistant \\
\hline
\end{tabular}

* Refer to Table 1

insect pests could be avoided.

\section{Acknowledgement}

Sincere thanks go to Prof. S. Nakata and Mr K. Komi both of the Faculty of Agriculture, Kochi University, Japan, for provision of chemicals and assistance in biochemical analyses of samples.

\section{References}

Devonshire, A. L. \& Moores, G. D. (1982) A carboxylesterase with broad substrate specificity causes organophosphorus and pyrethroid resistance in peach-potato aphids (Myzus persicae). Pestic Biochem. Physiol. 18, 235-246.

Devonshire, A. L. (1975) Studies of the carboxylesterases of Myzus persicae resistant and susceptible to organophosphorus insecticides. Proceedings of the $8^{\text {th }}$ British Insect. and Fung. Conf. pp. 67-73.

HAMA, H. \& Hosoda, A. (1988) Individual variations of aliesterase activity in field populations of Aphis gossypii Glover (Homoptera: Aphididae). Appl.Entomol. Zool. 23, 109-112.

Owusu, E. O. (1997) Preliminary biochemical evidence of insecticide resistance development in Ghanaian populations of cotton aphid, Aphis gossypii Glover (Homoptera: Aphididae). Ghana J. Sci. 37, 67-70.

Owusu, E. O., Horitke, M. \& Hirano, C. (1996a) Polyacrylamide gel electrophoretic assessment of esterases in cotton aphid (Homoptera: Aphididae) resistance to dichlorvos. J. econ. Entomol. 89, 302306.

Owusu, E. O., Horime, M. \& Hirano, C. (1996b) Inhibition of insecticides by partially purified carboxylesterase from Aphis gossypii (Homoptera: Aphididae). J. econ. Entomol. 89, 307-310.

Owusu, E. O., Komi, K., HoriIKe, M. \& Hirano, C. (1994) Some properties of carboxylesterase from Aphis gossypii Glover (Homoptera: Aphididae), Appl. Entomol. Zool. 29, 47-53.

Owusu, E.O. \& AdJeI-AfriyIE, F. (1996) Relationship 
between permethrin susceptibility and elevated esterase activities in cotton aphid (Homoptera: Aphididae) clones. Ghana J. Sci. 31-36, 95-99.

Owusu, E. O., Kim, C-S. \& HoritKe, M. (1995) Susceptibility of cotton aphid, Aphis gossypii Glover (Homoptera: Aphididae), clones to dichlorvos and its relationship to activity levels of some esterases.
Res. Rep. (Agric.) Kochi Univ. 44, 59-67.

YeBOAн, P. M. (2001) Variation of insecticide resistance development among species of aphid (Homoptera: Aphididae). (BSc. Dissertation.) Department of Zoology, Faculty of Science, University of Ghana. $60 \mathrm{pp}$.

Received 13 May 05; revised 06 Feb 07. 